\title{
BIBLIOGRAPHY
}

1. K. K. Chen, On the Cesarro-summability of the Laplace's series of hyperspherical functions, The Science Reports of the Tôhoku Imperial University vol. 17 (1928) pp. 1073-1089.

2. A. Erdélyi, W. Magnus, F. Oberhettinger, F. G. Tricomi, Higher transcendental functions, vol. 1, New York, 1953.

3. - Higher transcendental functions, vol. 2, New York, 1953.

4. G. H. Hardy, Divergent series, Oxford, 1949.

5. G. H. Hardy and J. E. Littlewood, Abel's theorem and its converse, Proc. London Math. Soc. vol. 18 (1920) pp. 205-235.

6. E. Kogbetliantz, Recherches sur la sommabilité des sérics ultrasphcriqucs par la méthod des moyennes arithmetiques, Jour. de Math. vol. 3 (1924) pp. 107-187.

7. G. Szegö, Orthogonal polynomials, New York, 1939.

8. A. Zygmund, Trigonometrical series, Warsaw, 1935.

RUTGERS UNIVERSITY,

New Brunswick, N. J.

The Institute for Advanced Stuis,

Princeton, N. J.

\section{ERRATA, VOLUME 79}

Arithmetical predicates and function quantifiers. By S. C. Kleene. Pages 312-340.

Page 329, lines 20-21. For "those with superscript "Q" partial recursive, uniformly in $Q$ );" read "those with superscript "Q" partial recursive uniformly in $Q) ; "$. 